\title{
The Activity of Gram-Positive Produced Lantibiotics against MRSA
}

\begin{abstract}
There is no doubt that the emergence and wide geographic spread of multidrugresistant bacteria has initiated a search for alternative new chemotherapeutic agents that would effectively fight against them. One class of agents that has recently received an increasing attention in research centers as a new line of therapy are the basic polypeptides, a class of bacteriocins called lantibiotics, which are naturally produced by some strains of bacteria and fight against other competing microbes residing in the same niche. Moreover, some novel strains of Gram-positive bacteria have particularly attracted the focus of many researchers as unique lantibiotics producers. It worth noting that not only do lantibiotics have unique features, biochemical structures and mode of actions, but they also have not yet been used in the setting of chemotherapy on the same scale as traditional antibiotics. This mini-review article will shed light on some lantibiotics that have potential as chemotherapeutic agents in the treatment of multi-drug resistant Staphylococcus aureus.
\end{abstract}

Keywords: Chemotherapeutic; Lantibiotics; Antibiotics; Gram-positive

Molume 5 Issue 3 - 2017
Mohammed Al Mahrous*
Department of Medical Microbiology, Maternity and Children
Hospital (Dammam), Saudi Arabia
*Corresponding author: Mohammed Al Mahrous, Medical
Microbiology Department, Laboratory Services, Maternity
and Children Hospital (Dammam), The Ministry of Health,
P.O.Box 60734, Qatif 31911, Eastern Province, Kingdom of
Saudi Arabia, Tel: 00966592334721;
Email: MAl-Mahrous@moh.gov.sa
Received: April 26, 2017 | Published: July 03, 2017

\section{Mini Review}

Hospital strains of Staphylococcus aureus are usually resistant to a variety of different antibiotics. A few strains are resistant to virtually all clinically useful antibiotics except vancomycin, and vancomycin-resistant strains have been reported in recent years. This resistance of staphylococcal strains to conventional antibiotics used to treat staphylococcal, in general, and MRSA infections, in particular, has reached an alarming level [1-3]. A contributing factor has been the widespread and/or inappropriate use of antibiotics to treat non-life-threatening infections that has generated a strong evolutionary pressure for the emergence and wide geographic spread of multidrug-resistant $S$. aureus that have resistance to particular groups of antibiotics. Thus, there is a pressing need to discover and develop new agents that are active against such resistant strains [3-6].

The recent strategy for finding new classes of antibacterial compounds based on targets identified from bacterial genomics has not yet proved successful. This has led to renewed interest in natural products, which have historically been invaluable as a source of antibacterial drugs, such as penicillin and glycopeptides, reflecting their evolutionary origin as "weapons" used by bacteria againsteach other. Glycopeptides were the first discovered example of peptides that kill bacteria by targeting lipid II, a bacteriaspecific membrane component that is essential in bacterial cellwall synthesis [7]. It has recently been demonstrated that lipid II is the target of several other natural products, including some classes of bacteriocins [5-7].

In recent years, there has been much focus on a promising class of bacteriocins known as lantibiotics [4]. It can be said that lantibiotics are antimicrobial peptides produced by some bacteria and are generally active against other bacteria belonging to the same or closely-related species. Gram-positive bacteria have attracted the focus of many researchers as producers of class-I bacteriocins, i.e. lantibiotics. The most prominent representative of lantibiotics, nisin, already has a long history of use in the protection of foodstuffs. Lantibiotics have also been considered for application in humans; however, they have not yet been used in the setting of chemotherapy on the same scale as traditional antibiotics [4].

There are two major groups of bacteriocins: those produced by gram-negative bacteria and those that are formed by gram-positive bacteria. The bacteriocins of gram-negative bacteria were the first to be studied extensively. The colicins, produced by members of the Enterobacteriaceae, are considered the prototypes. Many of the colicins have been extensively characterized, resulting in comprehensive knowledge of the genetic basis, domain structure, mode of formation, and killing action of these molecules. The colicins are large proteinaceous compounds with domains specific for certain functions, such as binding to receptor proteins in the outer membrane, translocation through the peptidoglycan layer and periplasmic space, and toxic activity which usually resides in the domain closest to the C-terminus of the molecule $[4,8]$.

The bacteriocins of gram-positive bacteria have been classified into numerous subtypes $[4,9,10]$ the principal being: (1) lantibiotics, (2) bacteriocins containing cysteine ring structures instead of lanthionine (Lan) and â-methyl-lanthionine (âMeLan), (3) bacteriocins whose mode of action is dependent on their content of a single cysteine (Cys) residue, (4) bacteriocins not containing free Cys residues and (5) bacteriocins whose action is dependent on the co-operative action of more than one peptide. Some of these subtypes have been further divided on the basis 
of characteristics (Table 1) such as their antimicrobial activity against important pathogenic bacteria, sometimes on the

assumption [with little or no evidence [8]] that similar activities are attributable to similar peptide sequences $[4,10]$.

Table 1: The proposed 'Universal' bactriocins classification [modified from [11]].

\begin{tabular}{|c|c|}
\hline I. Lantibiotics & II. Non-Lantibiotics \\
\hline Ia. Linear & Ila. Pediocin-like \\
\hline Ib. Globular & IIb. Miscellaneous \\
\hline Ic. Multicomponent & IIc. Multicomponent \\
\hline III. Large Protiens & IV. Cyclic Peptides or Complex Proteins \\
\hline IIIa. Bacteriolytic & IVa. Carbohydrate-associated protein \\
\hline IIIb. Non-lytic & IVb. Lipid-associated protein \\
\hline
\end{tabular}

A feature common to all of the gram-positive bacteriocin subtypes is that they are produced ribosomally as a prepeptide consisting of two parts or pseudo domains: the leader region and the propeptide component. After synthesis of the prepeptide, any amino acid modifications that may be necessary for the biological functionality of the peptide occur. These modifications appear to be restricted to the propeptide region. Then, during or after transportation from the cell, the leader region is proteolytically cleaved from the propeptide part, thus activating the bacteriocin $[4,9,10]$. It is this mechanism of ribosomal biosynthesis, sometimes followed by post translational modification, that principally distinguishes the bacteriocins from other peptide antibiotics (i.e. those produced in bacterial cells by multi-enzyme complexes) for which no gene encoding a ribosomally-synthesized peptide exists $[4,8]$.

Most bacteriocins carry a net positive charge at neutral $\mathrm{pH}$ with their biological activity often increasing with decreasing $\mathrm{pH}$. This is thought to be due to a decreased affinity of the bacteriocin for the cell wall components of susceptible bacteria, leaving more bacteriocin free to interact with the cytoplasmic membrane [9]. The mode of action of the majority of bacteriocins of gram-positive bacteria is thought to be a specific increase in permeability of the cytoplasmic membrane of sensitive cells through the formation of hydrophilic pores. These pores allow the uncontrolled efflux from the cell of certain essential intracellular components and also usually cause the dissipation of the proton motive force or proton gradient that is essential for the production of ATP $[4,9,10]$ (Figure 1).

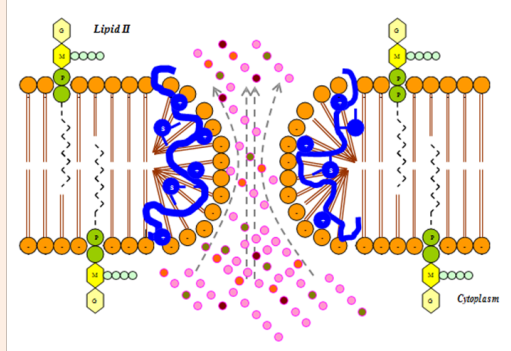

Figure 1: The proposed mechanism of action of lantibiotics in which hydrophilic pores are formed as a result of the attraction that takes place between the positive charge on lantibiotic and negative charge on lipid II of the cell membrane.
Since lantibiotics are generally produced by/and inhibit related organisms, Gram-positive bacteria in general and Staphylococcus spp. in particular are investigated by research centers for inhibitory activity against the highly resistant strains of $S$. aureus, especially epidemic-MRSA. These approaches are expected to give the opportunity for new therapeutic agents which can be used to combat the world widespread and threat of staphylococcal infections caused by MRSA, or even VRSA. Last and not least, lantibiotics have significant commercial value and broad applicability and practical methods for their production would have a significant economic impact [4].

\section{References}

1. Klein E, Smith DL, Laxminarayan R (2007) Hospitalizations and Deaths Caused by Methicillin-Resistant Staphylococcus aureus, United States, 1999-2005. Emerg infect dis 13(12): 1840-1846.

2. Kong E, Johnson J, Jabra Rizk M (2016) Community-Associated Methicillin-Resistant Staphylococcus aureus: An Enemy amidst Us. PLoS Pathog 12(10): e1005837.

3. Jury F, Al Mahrous M, Apostolou M, Sandiford S, Fox A, et al. (2006) Rapid cost-effective subtyping of meticillin-resistant Staphylococcus aureus by denaturing HPLC. J Med Microbiol 55(Pt 8): 1053-1060.

4. Al Mahrous M, Upton M (2011) Discovery and development of lantibiotics; antimicrobial agents that have significant potential for medical application. Expert Opin Drug Discov 6(2): 155-170.

5. Al Mahrous M, Jack RW, Sandiford SK, Tagg JR, Beatson SA, et al. (2011) Identification of a haemolysin-like peptide with antibacterial activity using the draft genome sequence of Staphylococcus epidermidis strain A487. FEMS Immunol Med Microbiol 62(3): 273-282.

6. Al Mahrous M, Sandiford SK, Tagg JR, Upton M (2010) Purification and characterization of a novel delta-lysin variant that inhibits Staphylococcus aureus and has limited hemolytic activity. Peptides 31(9): 1661-1668.

7. Breukink E, Kruijff B (2006) Lipid II as a target for antibiotics. Nat Rev Drug Discov 5(4): 321-323.

8. Jack Ralph W, Sahl HG, Bierbaum G (1998) Lantibiotics and related peptides. Springer, London, UK, pp. 224.

9. Jack RW, Tagg JR, Ray B (1995) Bacteriocins of Gram-positive bacteria. Microbiol Rev 59(2): 171-200.

10. Klaenhammer TR (1993) Genetics of bacteriocins produced by lactic acid bacteria. FEMS Microbiol Rev 12(1-3): 39-85. 
11. Nelson M, Patrick G, Gallagher P (2012) Methicillin-Resistant Staphylococcus aureus in the Neonatal Intensive Care Unit. Semin Perinatol 36(6): 424-430. 